\title{
Prenatal measurement of cardiothoracic ratio in evaluation of heart disease
}

\author{
D Paladini, S K Chita, L D Allan
}

\begin{abstract}
The cardiothoracic ratio was measured in $\mathbf{4 1 0}$ normal fetuses and in a group of 73 fetuses with functional or structural heart disease. In normal fetuses it was fairly constant throughout pregnancy, but of those with congenital heart disease it was raised in cases of Ebstein's anomaly, tricuspid dysplasia, atrioventricular septal defect, and complete heart block. In some forms of congenital heart disease, however, it was within the normal range. There was a significant positive correlation between the cardiothoracic ratio and fetal hydrops in the group of 15 fetuses with supraventricular tachycardias. In these fetuses the cardiac size decreased significantly once the fetus reverted to sinus rhythm after the mother had been treated. Measurement of the cardiothoracic ratio is essential in the evaluation of fetal hydrops, as an increased value may point to the diagnosis of an intermittent fetal tachycardia if the fetus is assessed during a period of sinus rhythm.

The measurement of this index forms a part of the complete prenatal evaluation of structural heart disease. The degree of cardiomegaly may provide useful information about secondary lung compression or cardiac failure and therefore assist in giving an accurate prognosis for postnatal survival.
\end{abstract}

The ratio of the size of the heart to that of the thorax on a plain chest radiograph has long been used as an index of cardiac disease or heart failure in both paediatric and adult cardiology. Many forms of congenital heart disease, however, do not show cardiac enlargement but have abnormal measurements of individual chambers. For that reason cardiologists evaluating the fetal heart have measured the ventricular or atrial size by echocardiography throughout gestation to provide a normal range of measurements for comparison with those with suspected malformations. ${ }^{12}$ The cardiothoracic ratio has been assessed in normal patients ${ }^{3}$ and used to evaluate pulmonary volume prenatally in thoracic hypoplasia, but this measurement has not to our knowledge been used to assess the heart in the presence of structural malformation. ${ }^{4}$ For this reason, we measured the cardiothoracic ratio throughout pregnancy in normal fetuses and in groups of fetuses with specific functional or structural heart disease.

Subjects and methods

Since the beginning of 1985 we have examined more than 2000 pregnancies. All the echocardiograms have been recorded on videotape. The records of 410 examinations, selected retrospectively, have been used to acquire the normal data for the cardiothoracic ratio. The selection criteria for these pregnancies were: that the gestational age had been assessed by early ultrasound examination, the pregnancy was uneventful, the neonatal outcome was normal, there was a suitable thoracic view for measurement on the videotape record, and there was no sign of congenital heart disease.

A Hewlett-Packard 77020A sector scanner with 5.0 and $3.5 \mathrm{mHz}$ probes was used. The thoracic view was considered suitable for measurement when the whole thorax was seen on the screen, a good four chamber view was achieved, and there was a complete rib and no abdominal contents in the frame. All the mesurements were performed in diastole (fig 1). The circumference of the heart and the circumference of the thorax were measured and ex-
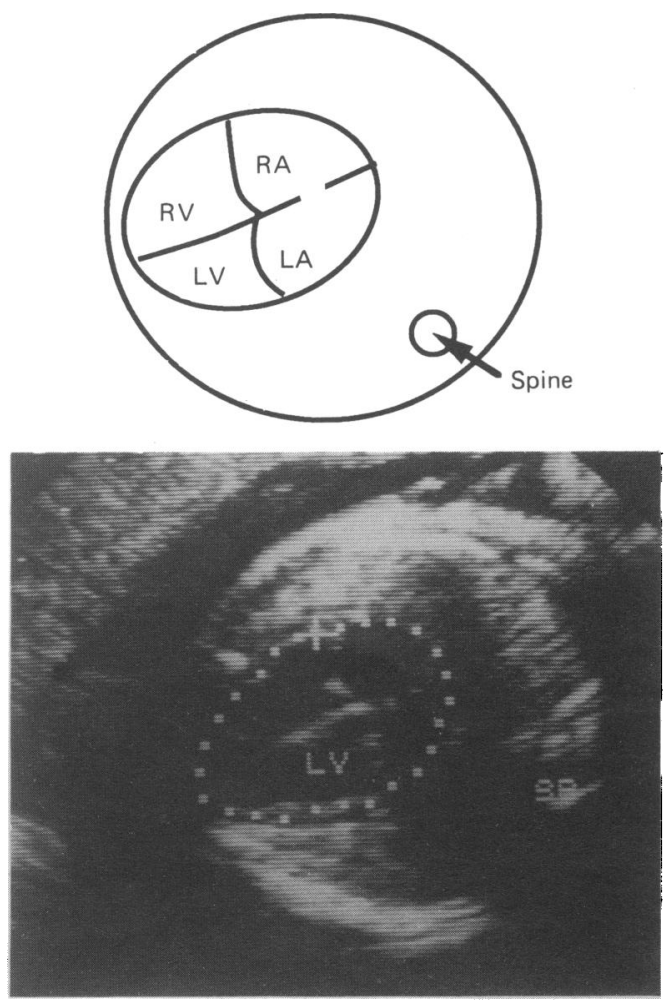

Figure 1 A thoracic section suitable for measurement. The cardiac circumference is indicated. $R A=$ right atrium, $L A=$ left atrium, $L V=$ left ventricle, and $R V=$ right ventricle. 


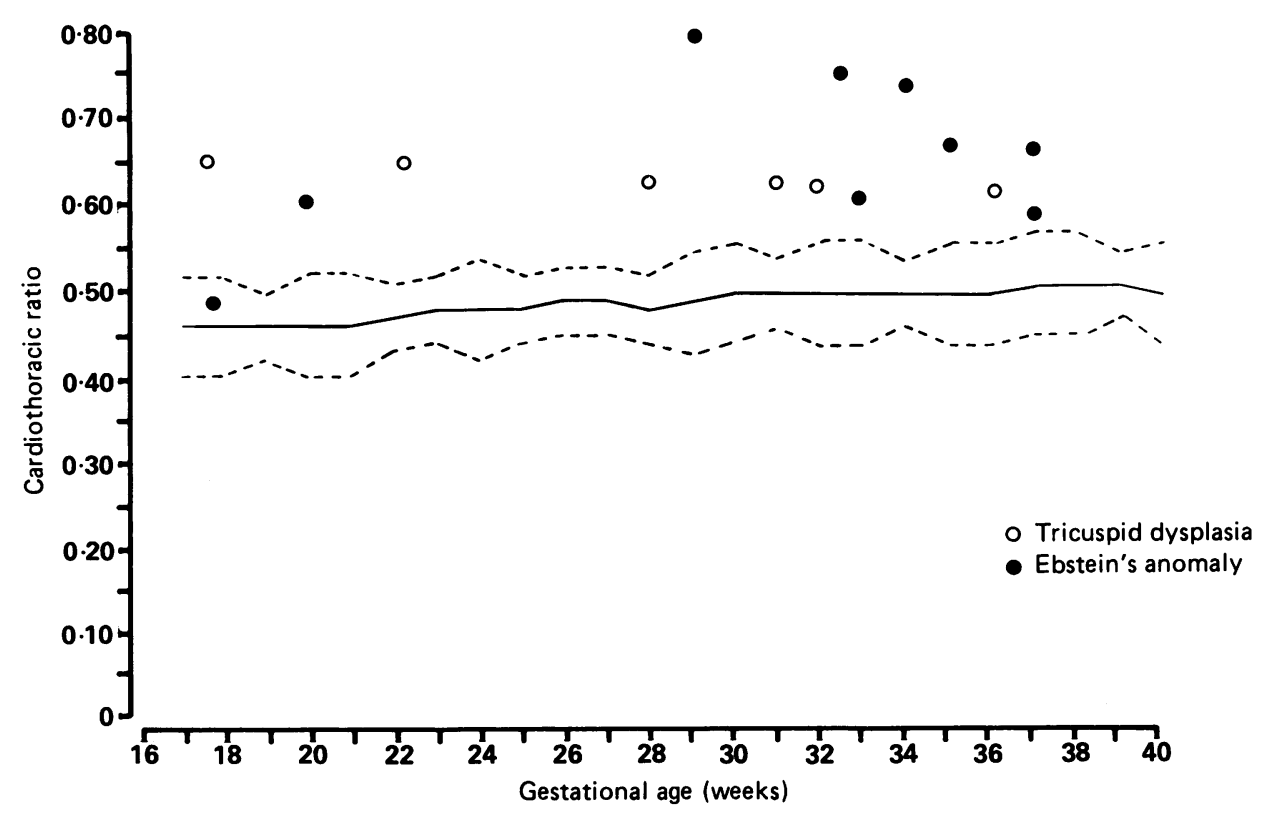

Figure 2 The cardiothoracic ratio in 15 cases of tricuspid valve abnormality compared with the normal range.

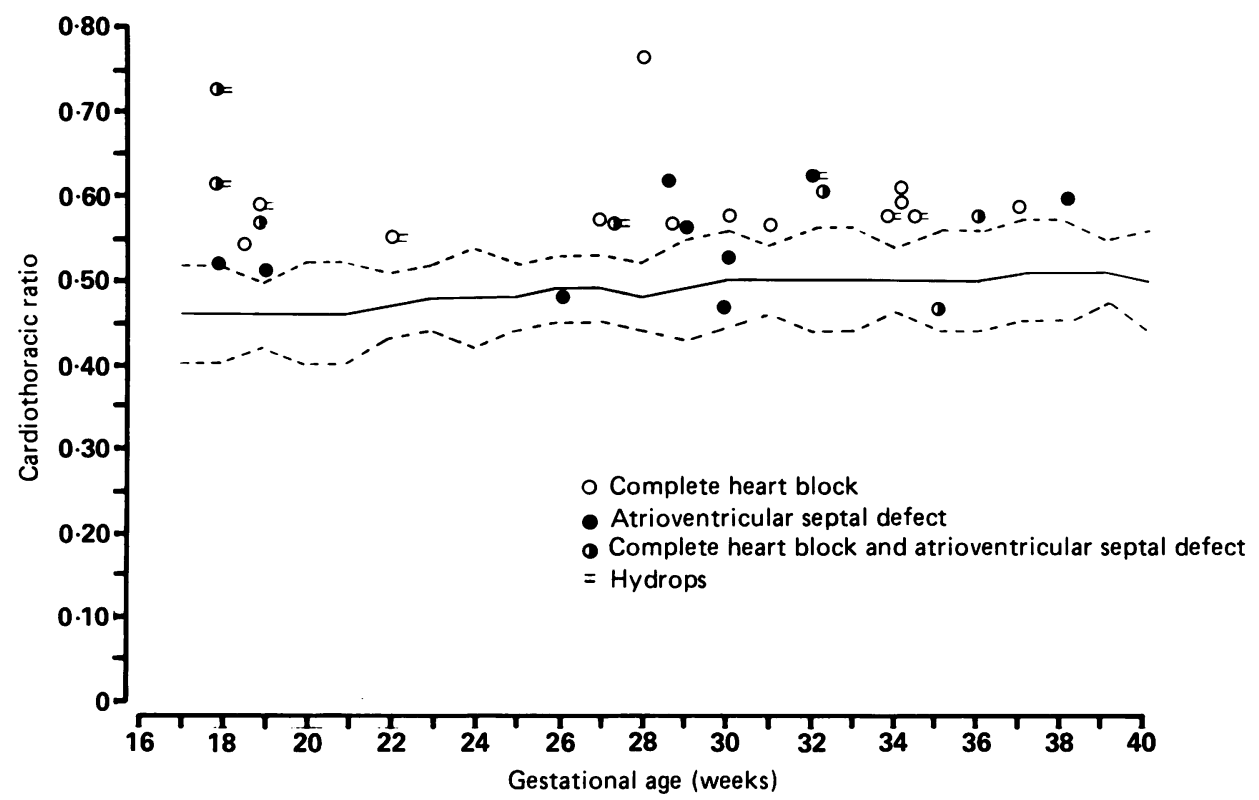

Figure 3 With only one exception, all cases of complete heart block showed cardiomegaly on the cardiothoracic ratio whether or not there was associated fetal hydrops.

pressed as a ratio. The mean was taken of two different measurements in two different frames.

Four groups of fetuses were studied including 15 with Ebstein's anomaly or tricuspid dysplasia, 29 with complete heart block, or an atrioventricular septal defect, or both, 15 with atrial tachycardias, and a further 14 with various forms of congenital heart disease.

Statistical analysis was by Student's nonpaired two tailed $t$ test.

\section{Results}

The cardiothoracic ratio was fairly constant throughout pregnancy, with a slight increase from 0.45 at 17 weeks to 0.50 at term. The normal range with $95 \%$ confidence interval is indicated in the graphs of the abnormal groups (figs 2 to 5 ).

In the cases with tricuspid valve abnormality, there was a high cardiothoracic ratio in 14 of 15 cases (fig 2). One case with a mild form of Ebstein's anomaly seen in early pregnancy was within the normal range.

Within the second group there were 13 cases of isolated complete heart block, nine cases of atrioventricular septal defect, and seven with both an atrioventricular septal defect and complete heart block (fig 3). The cardiothoracic ratio was increased in 24 fetuses. In all the cases 


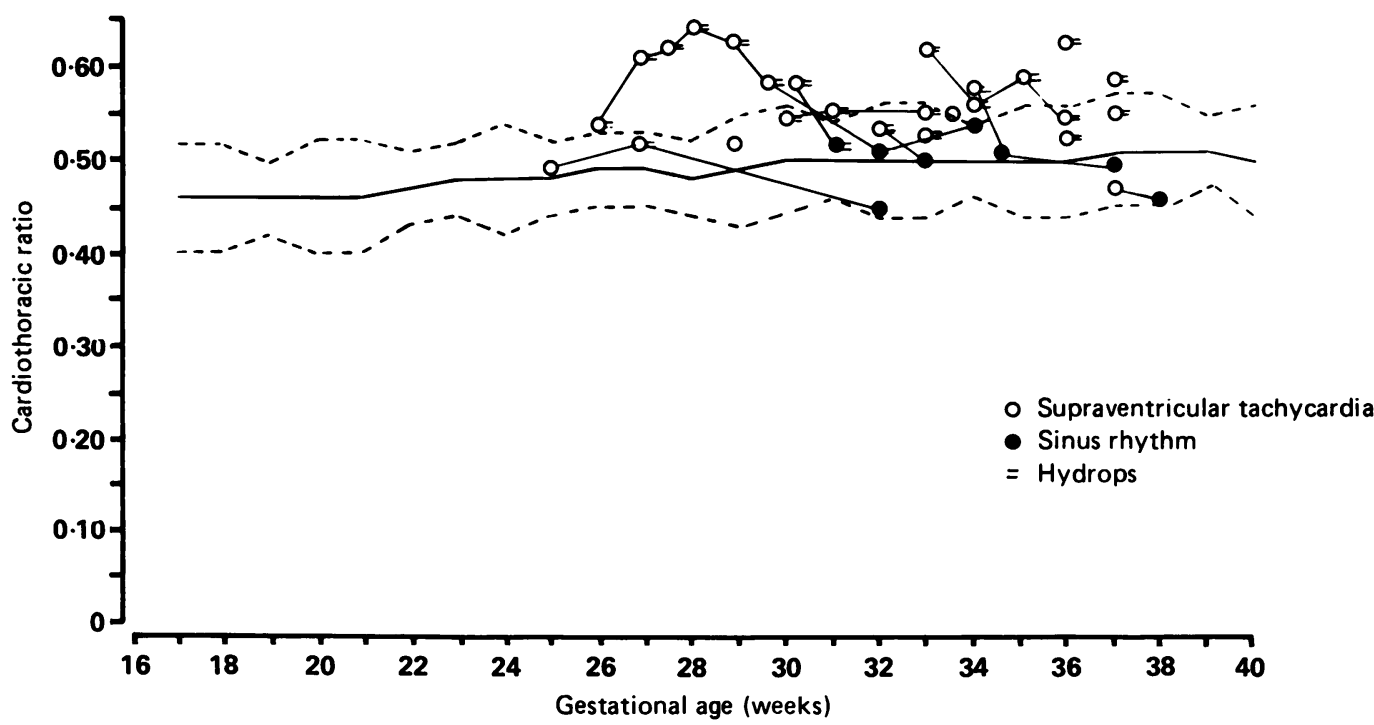

Figure 4 Serial measurement of the cardiothoracic ratio in cases of fetal tachycardia showing correlation between the presence of hydrops and cardiac enlargement. Some fetuses remained hydropic after conversion to sinus rhythm and after the cardiothoracic ratio became normal.

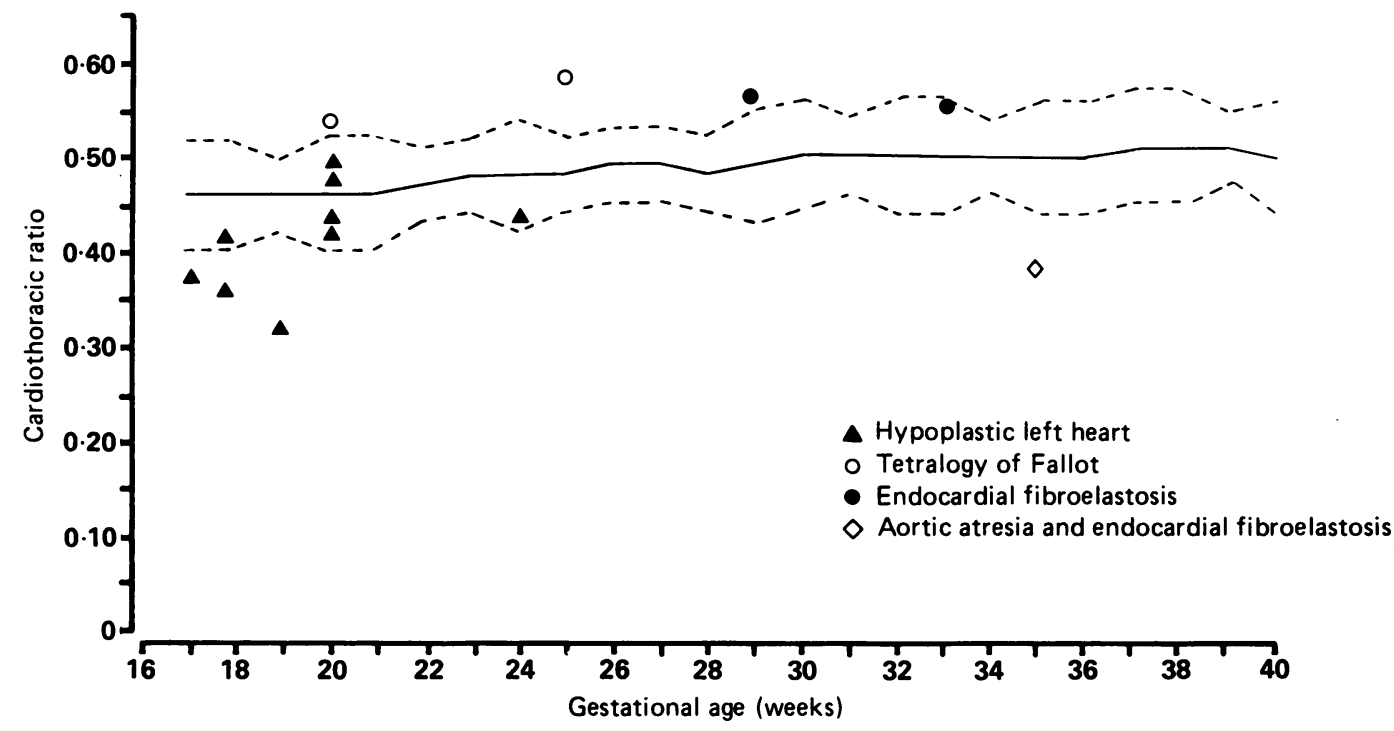

Figure 5 An assorted group of cases of fetal heart disease compared with the normal range. No consistent pattern of cardiothoracic ratio measurement was found.

complicated by fetal hydrops the ratio was increased, but there was no clear difference in cardiac size between the hydropic and nonhydropic cases with complete heart block.

In those cases with a fetal tachycardia, 34 measurements were made (fig 4). Not all those complicated by fetal hydrops were above the normal range, but the mean (SD) cardiothoracic ratio for the hydropic fetuses was $0.55(0.34)$ against $0.49(0.3)$ for the non-hydropic cases $(p<0.01)$. Nine measurements were made after the fetuses had reverted to sinus rhythm because the mother was receiving treatment. In these cases, the ratios fell significantly $(p<0.01)$ from the estimates before treatment $(0.50(0.28)$ compared with $0.55(0.37))$.

In the last group cases with different forms of congenital heart disease were evaluated (fig 5).

No clear pattern of cardiac size is characteristic of all forms of congenital heart disease.

\section{Discussion}

A suitable view for estimation of the cardiothoracic ratio can readily be obtained in all patients. Some forms of heart disease are particularly associated with cardiac enlargement-for example, Ebstein's anomaly and tricuspid dysplasia. This form of heart disease is commonly associated with early neonatal death from lung hypoplasia. The underdevelopment of the lungs occurs as a result of lung compression, which is secondary to often gross cardiomegaly. It is possible that the degree of cardiac 
enlargement in these cases may correlate with the postnatal outcome. Expansion of these data may answer this.

There are some forms of heart disease where the heart is not enlarged prenatally, or is below the normal range of cardiac size-for example, hypoplastic left heart syndrome. In all cases but one in complete heart block the heart was enlarged, whether or not there was associated fetal hydrops.

Where cardiac failure develops as a result of an intrauterine tachycardia, obvious cardiac enlargement is detected by measurement of the cardiothoracic ratio. A fetus can remain hydropic, however, after a supraventricular tachycardia has been controlled and the heart size has returned to normal. This may indicate that the fetus has difficulty in clearing peripheral fluid collections even after cardiac function has returned to normal.
In summary, measurement of the cardiothoracic ratio is simple to do and should be part of the complete prenatal evaluation of structural or functional heart disease. The degree of increase of this index from the normal range may assist in predicting the extent of lung compression and the severity of heart failure.

DP was on secondment from the University of Naples, and SKC and LDA were supported by the British Heart Foundation.

1 Allan LD, Joseph MD, Boyd EGCA, Campbell S, Tynan MJ $M$ mode echocardiography in the developing human fetus

2 De Vore GR, Donnerstein RL, Kleinman CS. Fetal echocardiography II. The diagnosis and significance of a pericardial effusion in the fetus using real-time-directed M-mode ultrasound. Am $\mathcal{F}$ Obstet Gynecol 1982;144:693-701.

3 Jordaan HVF. Cardiac size during prenatal development. Obstet Gynecol 1987;69:854-8.

4 De Vore GR, Horenstein J, Platt LD. Fetal echocardiography. VI. Assessment of cardiothoracic disproportion-a new technique for the diagnosis of thoracic hypoplasia. $A m \mathcal{F}$ Obstet Gynecol 1986;155:1066-71. 